Fernando Zegarra Sánchez

\title{
Ignição por Compressão de Etanol Aditivado em uma Máquina de Compressão Rápida
}

Tese apresentada ao Programa de Pós-Graduação em Engenharia Mecânica da PUC-Rio como requisito parcial para obtenção do título de Doutor em Engenharia Mecânica

Orientador : Prof. Carlos Valois Maciel Braga Co-Orientador: $\quad$ Prof. Sergio Leal Braga 
Fernando Zegarra Sánchez

\section{Ignição por Compressão de Etanol Aditivado em uma Máquina de Compressão Rápida}

Tese apresentada como requisito parcial para obtenção do grau de Doutor pelo Programa de Pós-Graduação em Engenharia Mecânica do Programa de Pós-Graduação da PUC-Rio. Aprovada pela Comissão Examinadora abaixo assinada.

Prof. Carlos Valois Maciel Braga

Orientador

Departamento de Engenharia Mecânica - PUC-Rio

Prof. Sergio Leal Braga

Co-Orientador

Departamento de Engenharia Mecânica - PUC-Rio

Profa. Ana Rosa Fonseca de Aguiar Martins

Departamento de Engenharia Química e de Materiais - PUC-Rio

Prof. Marcos Sebastião de Paula Gomes

Departamento de Engenharia Mecânica - PUC-Rio

Dr. Ricardo Hernandez Pereira General Electric Company

Dr. Guilherme Bastos Machado Cenpes/Petroleo Brasileiro S.A. - PETROBRAS

Prof. Márcio da Silveira Carvalho

Coordenador Setorial do Centro Técnico Científico - PUC-Rio

Rio de Janeiro, 29 de Setembro de 2016 
Todos os direitos reservados. É proibida a reprodução total ou parcial do trabalho sem autorização da universidade, do autor e do orientador.

\section{Fernando Zegarra Sánchez}

Graduou-se em Engenharia Mecatrônica na Universidad Nacional de Ingeniería, Lima - Peru, em Julho de 2007. Desde 2007 até 2010 atuou como engenheiro de projetos e manutenção em diversas firmas peruanas, na área de subestações elétricas e maquinaria para mineração. obteve o Título de Mestre em Engenharia Mecânica na PUC - Rio em 2012. Atualmente é engenheiro de pesquisa no LEV/PUC-Rio, estando envolvido no uso de combustíveis alternativos para motores de combustão interna.

Ficha Catalográfica

Sánchez, Fernando Zegarra

Ignição por Compressão de Etanol Aditivado em uma Máquina de Compressão Rápida / Fernando Zegarra Sánchez; orientador: Carlos Valois Maciel Braga; co-orientador: Sergio Leal Braga. - Rio de Janeiro PUC-Rio, Departamento de Engenharia Mecânica, 2016.

v., 190 f: il. (color) ; $30 \mathrm{~cm}$

Tese (doutorado) - Pontifícia Universidade Católica do Rio de Janeiro, Departamento de Engenharia Mecânica.

Inclui referências bibliográficas.

1. Engenharia Mecânica - Tese. 2. Mistura de Etanol e n-Butanol;. 3. Etanol Aditivado;. 4. Ciclo de Ignição por Compressão;. 5. Combustíveis Renováveis.. I. Braga, Carlos Valois Maciel. II. Braga, Sérgio Leal. III. Pontifícia Universidade Católica do Rio de Janeiro. Departamento de Engenharia Mecânica. IV. Título. 
Dedico este trabalho a minha mãe Berta, a meu tio Lidio e a toda minha família, pelo imenso amor e apoio desde meus primeiros anos de vida. 


\section{Agradecimentos}

Agradeço a Deus por tudo na minha vida. Pela força, luz e determinação na escolha da direção certa. Agradeço a Ele todas as vitórias e conquistas alcançadas.

A minha familia pelo amor, apoio incondicional, compreensão, sacrifício, incentivo, felicidade, carinho, dedicação e inspiração encontrada na minha querida família que sempre farão parte de cada luta e vitória.

Ao Professor Carlos Valois Maciel Braga, meu orientador e amigo, pelo qual tenho muita estima e admiração. Muito obrigado pelas idéias e pelas inúmeras conversas esclarecedoras em torno do trabalho e pelas revisões no decorrer da redação do presente manuscrito

Ao Professor Sergio Leal Braga, meu co-orientador e também amigo, pela confiança e parceria para a realização deste trabalho. Agradeço por ter acreditado no meu potencial e por todas as oportunidades que me deu. Muito obrigado pelo acompanhamento, motivação, revisão e sugestões na redação do presente trabalho. Para mim foi um grande prazer poder trabalhar ao seu lado.

Ao Departamento de Engenharia Mecânica por seu apoio durante todos estes anos.

Agradecimentos em particular para os órgãos de fomento à pesquisa CAPES, pelo apoio financeiro, sem o qual este trabalho simplesmente não teria sido possível.

A PSA Peugeot Groupe, pelo suporte financeiro e auxílios concedidos para o desenvolvimento deste trabalho.

Ao Instituto Tecnológico, ITUC/PUC-Rio, pelo suporte com a construção de equipamentos e a logística para a compra de insumos e instrumentação para a Sala de Testes de Motores do Laboratório de Engenharia Veicular, LEV/PUC-Rio.

Aos colegas do LEV/PUC-Rio, Giovanni Calfa Neto e Leonardo Costa Braga pelo companheirismo e continuado incentivo; Severino Wanderley e Nestor Corrêa Cotelo pelo suporte eletrônico e importantes contribuições; Gilson Coutinho Pradanoff, Jorge Ricardo Moura Junior e Bruno Alves Maia pelo auxilio na montagem do aparato experimental; Gerson Silveiro pela assistência na manutenção dos equipamentos. 


\section{Resumo}

Sánchez, Fernando Zegarra; Braga, Carlos Valois Maciel; Braga, Sérgio Leal. Ignição por Compressão de Etanol Aditivado em uma Máquina de Compressão Rápida. Rio de Janeiro, 2016. 190p. Tese de Doutorado - Departamento de Engenharia Mecânica, Pontifícia Universidade Católica do Rio de Janeiro.

Com o passar do tempo, a humanidade tem uma maior dependência na geração de energia, utilizada para promoção de conforto, transporte e outros. Com a finalidade de resolver este aumento de demanda, novas fontes eficientes, de preferência renováveis, estão sendo pesquisadas. O transporte é uma das atividades que tem maior dependência dos combustíveis fósseis, além de ser também um dos maiores geradores de gases de efeito estufa. É por isso, que em diversas partes do mundo, o homem pesquisa novas fontes de energia renováveis que possam ser substitutas dos atuais tradicionais usados no transporte. Sabe-se, que os motores Diesel são mais eficientes com relação aos motores Otto. Devido a este fato, há mais de 30 anos pesquisam-se e desenvolvem-se sistemas de ignição por compressão, movidos com combustíveis renováveis, o qual permita a diminuição da dependência dos combustíveis fósseis e garanta a redução de gases de efeito estufa. O etanol é um candidato para substituir o óleo Diesel, mas tem que se levar em conta algumas alterações (aumento da relação de compressão, adição de melhoradores da autoignição, etc.) antes de ser utilizado nos motores Diesel. Com base nisto, a presente tese apresenta uma nova proposta, utilizar como melhorador da autoignição do etanol o n-butanol. Para tal propósito se desenvolveu diversos testes com diversas relações de compressão, percentuais em massa de aditivo na mistura de etanol e diversos avanços da injeção. Os testes foram realizados em uma máquina de compressão rápida (MCR) com misturas de etanol e polietilenoglicol 400 e 600, e n-butanol, além dos testes referenciais com óleo Diesel e ED95. Os resultados mostram que o nbutanol, com uma participação de $10 \%$ na mistura, pode ser utilizado como melhorador da autoignição do etanol em sistemas de ignição por compressão.

\section{Palavras-chave}

Mistura de Etanol e n-Butanol; Etanol Aditivado; Ciclo de Ignição por Compressão; Combustíveis Renováveis. 


\section{Abstract}

Sánchez, Fernando Zegarra; Braga, Carlos Valois Maciel (Advisor); Braga, Sérgio Leal. Compression Ignition of Ethanol-Powered in Rapid Compression Machine. Rio de Janeiro, 2016. 190p. DSc. Thesis - Departamento de Engenharia Mecânica, Pontifícia Universidade Católica do Rio de Janeiro.

Over time, humanity has developed a greater reliance in power generation, used to promote comfort, transport and others. In order to address this increased demand new efficient sources are being searched, in preference, renewable sources. Transportation is one of the activities that have greater reliance on fossil fuels as well as being one of the largest generators of greenhouse gases. Therefore, in many parts of the world men are engaged in the search of new renewable energy sources that can substitute the current one used in transport. It is known that diesel engines are more efficient in comparison to the Otto engine. Due to this fact, for more than 30 years research has been conducted in order to develop ignition systems by compression, powered with renewable fuels, which reduces the dependence on fossil fuels and the emission of greenhouse gases. Ethanol is a viable candidate to replace diesel oil, but some improvements have to be accounted for before it's used in diesel engines, improvements such as the increase in compression ratio, adding auto-ignition improves, etc. Based on the facts presented, this thesis offers a new proposal, the use of n-butanol as an auto-ignition improver for ethanol. For this purpose several tests have been executed with various compression ratios, mass percentage of additive in the mixture of ethanol and many start of injections. The tests were performed in a rapid compression machine (RCM) with mixtures of ethanol and polyethylene glycol 400 and 600, and n-butanol in addition to the reference test with diesel oil and ED95. The results show that n-butanol with a $10 \%$ share of the mixture, can be used as an auto ignition improver for ethanol in compression ignition systems.

\section{Keywords}

Ethanol and n-Butanol Blend; Ethanol-Powered; Compression Ignition Cycle; Renewable Fuels. 


\section{Sumário}

1 Introdução $\quad 20$

1.1 Objetivos 28

1.2 Organização da Tese 28

2 Revisão da Literatura $\quad 30$

2.1 Ignição por Compressão 30

2.1.1 Detonação 32

2.1.2 Combustão Ideal $\quad 33$

2.1.3 Atraso da ignição 34

2.2 Uso de etanol em motores de ignição por compressão 37

2.2.1 Misturas etanol - diesel $\quad 40$

2.2.2 Emulsões etanol - diesel 41

2.2.3 Injeção de etanol no coletor de admissão ("fumigação") 43

2.2.4 Dupla Injeção Direta 47

2.2.5 Substituição total do óleo diesel mediante o uso de melhoradores da autoignição

2.3 Combustíveis e aditivos melhoradores da autoignição do etanol 50

2.3.1 Óleo Diesel 50

2.3.2 Etanol $\quad 52$

2.3.3 Etanol Aditivado - ED95 53

2.3.4 Misturas de Etanol e melhoradores da autoignição 57

2.3.5 Métodos de Modelagem 64

3 Aparato Experimental $\quad 67$

3.1 Bancada de Teste 68

$\begin{array}{lll}3.1 .1 & \text { MCR } & 69\end{array}$

$\begin{array}{lll}3.1 .2 & \text { Sistema Common Rail } & 74\end{array}$

$\begin{array}{lll}3.1 .3 & \text { Sistema de Injeção EFS } & 76\end{array}$

$\begin{array}{lll}\text { 3.1.4 Câmera de Alta velocidade } & 78\end{array}$

3.1.5 Sistema de Aquisição de Dados de Pressão 78

4 Modelagem Experimental e Procedimento de Cálculo $\quad 80$

4.1 Temperatura no Interior do Cilindro 84

4.2 Atraso da Ignição 85

$\begin{array}{lll}4.3 & \text { Testes na MCR } & 87\end{array}$

4.3.1 Testes preliminares $\quad 87$

$\begin{array}{lll}\text { 4.3.2 Testes finais } & 95\end{array}$

4.4 Tratamento dos Dados 97

5 Resultados e Discussões $\quad 99$

5.1 Resultados dos Testes Preliminares $\quad 99$

5.1.1 Testes de Diesel S50 e ED95 99

5.1.2 Testes de Misturas de Etanol com PEG400 108

5.1.3 Testes de Misturas de Etanol com PEG600 124 
5.1.4 Testes de Misturas Etanol com n-Butanol 132

5.2 Resultados dos Testes Finais 141

5.2.1 Testes com Diesel S10 141

5.2.2 Testes das misturas de etanol e PEG400 (13\%) 151

$\begin{array}{lll}5.2 .3 & \text { Testes das misturas de etanol e n-butanol (10\%) } & 158\end{array}$

$\begin{array}{lll}\text { 5.2.4 Comentários Finais } & 163\end{array}$

6 Conclusões e Recomendações $\quad 167$

$\begin{array}{ll}6.1 \text { Conclusões } & 167\end{array}$

6.1.1 Influências na utilização do combustível alternativo 167

6.1.2 Efeitos do avanço da injeção 170

6.2 Recomendações e Sugestões para Trabalhos Futuros 171

$\begin{array}{lr}\text { Referências bibliográficas } & 174\end{array}$

$\begin{array}{ll}\text { A Software de Analise de Dados } & 183\end{array}$

B Analise das Incertezas $\quad \mathbf{1 8 5}$

B.1 Análise na Medição de Variáveis $\quad 185$

B.2 Propagação das incertezas 186 


\section{Lista de figuras}

1.1 Emissões antrópicas totais associadas à matriz energética brasileira (2014), em $\mathrm{MtCO}_{2}$-eq.

1.2 Emissões brasileiras de gases de efeito estufa, período 1990-2012 em $\mathrm{CO}_{2}$ eq .

1.3 Ciclo termodinâmico do motor de ignição por compressão para a combustão de óleo diesel e etanol

2.1 Fases de combustão - ignição por compressão.

2.2 Atraso da ignição.

2.3 Etapas do atraso da ignição.

2.4 Composição do ED 95.

2.5 Fórmula molecular do polietileno glicol. 58

2.6 Cota do mercado em 2014 para n-butanol. 63

3.1 MCR instalada no LEV (PUC-Rio).

3.2 Adaptação e instalação da MCR para testes de ignição por compressão.

3.3 Desenho representativo do interior da MCR.

3.4 Posição do sistema no início do processo de compressão.

3.5 Ação do fluido da válvula de estrangulamento entre o pistão de equilíbrio mássico e a ação da válvula quando o pistão está próximo ou no PMS (Stop).

3.6 Posição do sistema no final do processo de compressão. $\quad 72$

3.7 Fotografias do cabeçote da MCR. $\quad 73$

3.8 Tela de interface do usuário na MCR(RCM CAMAS V3.3).

3.9 Vista em corte de uma bomba de alta pressão Bosch modelo CP3.3. 75

3.10 Acumulador de pressão e injetor instalado na bancada de teste. $\quad 76$

3.11 Injetor Common Rail (acima) e em corte (abaixo). 77

3.12 Injetor Common Rail instalado no cabeçote da MCR.

3.13 Sistema de injeção EFS e fotos da tela da interface do usuário no EFS.

3.14 Câmera de alta velocidade, esquema de filmagem dos ensaios e capturas do vídeo de combustão de diesel.

3.15 Sistema de aquisição de dados de pressão na bancada de teste.

4.1 Volume de controle considerando as válvulas fechadas na máquina de compressão rápida.

4.2 Determinação do início da combustão através da curva de calor liberado.

4.3 Determinação do início da combustão mediante a utilização das curvas de pressão e suas respectivas derivavas.

5.1 Pressão no cilindro para o teste com Diesel S50, $1500 \mathrm{rpm} \mathrm{e} \mathrm{RC} \mathrm{=}$ 15,92:1. 
5.2 Ignição e combustão no teste com diesel S50, $1500 \mathrm{rpm}, \mathrm{RC}=$ $15,92: 1$ e IDI $=2,40 \mathrm{~mm}\left(\sim 15^{\circ}\right.$ - Quadro 11$)$ antes do PMS. Imagens a 30.000 qps.

5.3 Pressão no cilindro para o teste com ED95, $1750 \mathrm{rpm}$ e RC $=20,65: 1.104$

5.4 Ignição e combustão no teste com ED95, $1750 \mathrm{rpm}, \mathrm{RC}=20,65: 1$ e IDI $=4,10 \mathrm{~mm}\left(\sim 20^{\circ}-\right.$ Quadro 8$)$ antes do PMS. Imagens a 30.000 qps.

5.5 Ignição e combustão no teste com ED95, 1750 rpm, RC = 20,65:1 e IDI $=6,20 \mathrm{~mm}\left(\sim 25^{\circ}-\right.$ Quadro 6$)$ antes do PMS. Imagens a 30.000 qps.

5.6 Pressão no cilindro para o teste com ED95, 1900 rpm e RC $=24,75: 1.106$

5.7 Ignição e combustão no teste com ED95, $1900 \mathrm{rpm}, \mathrm{RC}=24,75: 1$ e IDI $=2,40 \mathrm{~mm}\left(\sim 15^{\circ}\right.$ - Quadro 7$)$ antes do PMS. Imagens a 30.000 qps.

5.8 Ignição e combustão no teste com ED95, 1900 rpm, RC = 24,75:1 e IDI $=4,30\left(\sim 20^{\circ}\right.$ - Quadro 6) $\mathrm{mm}$ antes do PMS. Imagens a 30.000 qps.

5.9 Ignição e combustão no teste com ED95, $1900 \mathrm{rpm}, \mathrm{RC}=24,75: 1$ e IDI $=6,60 \mathrm{~mm}\left(\sim 24^{\circ}-\right.$ Quadro 5$)$ antes do PMS. Imagens a 30.000 qps.

5.10 Pressão no cilindro para o teste com a mistura de etanol e PEG400 $(13 \%), 1750 \mathrm{rpm}$ e $\mathrm{RC}=21,64: 1$.

5.11 Ignição e combustão no teste da mistura etanol e PEG400 (13\%), $1750 \mathrm{rpm}, \mathrm{RC}=21,64: 1$ e IDI $=4,70 \mathrm{~mm}\left(\sim 24^{\circ}\right.$ - Quadro 5) antes do PMS. Imagens a 30.000 qps.

5.12 Ignição e combustão no teste da mistura etanol e PEG400 (13\%), $1750 \mathrm{rpm}, \mathrm{RC}=21,64: 1$ e IDI $=6,10 \mathrm{~mm}\left(\sim 27^{\circ}\right.$ - Quadro 4$)$ antes do PMS. Imagens a 30.000 qps.

5.13 Ignição e combustão no teste da mistura etanol e PEG400 (13\%), $1750 \mathrm{rpm}, \mathrm{RC}=21,64: 1$ e IDI $=9,50 \mathrm{~mm}\left(\sim 33^{\circ}\right.$ - Quadro 5$)$ antes do PMS. Imagens a 30.000 qps.

5.14 Pressão no cilindro para o teste com a mistura de etanol e PEG400 $(13 \%), 1900 \mathrm{rpm}, \mathrm{RC}=25,88: 1$.

5.15 Ignição e combustão no teste da mistura etanol e PEG400 (13\%), $1900 \mathrm{rpm}, \mathrm{RC}=25,88: 1$ e IDI $=7,30 \mathrm{~mm}\left(\sim 24^{\circ}\right.$ - Quadro 5) antes do PMS. Imagens a 30.000 qps.

5.16 Ignição e combustão no teste da mistura etanol e PEG400 (13\%), $1900 \mathrm{rpm}, \mathrm{RC}=25,88: 1$ e IDI $=8,80 \mathrm{~mm}\left(\sim 27^{\circ}\right.$ - Quadro 5) antes do PMS. Imagens a 30.000 qps.

5.17 Ignição e combustão no teste da mistura etanol e PEG400 (13\%), $1900 \mathrm{rpm}, \mathrm{RC}=25,88: 1$ e IDI $=11,00 \mathrm{~mm}\left(\sim 31^{\circ}-\right.$ Quadro 5$)$ antes do PMS. Imagens a 30.000 qps.

5.18 Pressão no cilindro para o teste com a mistura de etanol e PEG400 $(15 \%), 1750 \mathrm{rpm}$ e $\mathrm{RC}=22,28: 1$.

5.19 Ignição e combustão no teste da mistura etanol e PEG400 (15\%), $1750 \mathrm{rpm}, \mathrm{RC}=22,28: 1$ e IDI $=2,60 \mathrm{~mm}\left(\sim 17^{\circ}-\right.$ Quadro 7$)$ antes do PMS. Imagens a 30.000 qps. 
5.20 Ignição e combustão no teste da mistura etanol e PEG400 (15\%), $1750 \mathrm{rpm}, \mathrm{RC}=22,28: 1$ e IDI $=4,90 \mathrm{~mm}\left(\sim 23^{\circ}\right.$ - Quadro 6) antes do PMS. Imagens a 30.000 qps.

5.21 Ignição e combustão no teste da mistura etanol e PEG400 (15\%), $1750 \mathrm{rpm}, \mathrm{RC}=22,28: 1$ e IDI $=5,80 \mathrm{~mm}\left(\sim 25^{\circ}\right.$ - Quadro 5) antes do PMS. Imagens a 30.000 qps.

5.22 Pressão no cilindro para o teste com a mistura de etanol e PEG400 $(15 \%), 1900 \mathrm{rpm}$ e RC $=25,58: 1$.

5.23 Ignição e combustão no teste da mistura etanol e PEG400 (15\%), $1900 \mathrm{rpm}, \mathrm{RC}=25,58: 1$ e IDI $=2,60 \mathrm{~mm}\left(\sim 17^{\circ}\right.$ - Quadro 7$)$ antes do PMS. Imagens a 30.000 qps.

5.24 Ignição e combustão no teste da mistura etanol e PEG400 (15\%), $1900 \mathrm{rpm}, \mathrm{RC}=25,58: 1$ e IDI $=5,00 \mathrm{~mm}\left(\sim 23^{\circ}\right.$ - Quadro 6) antes do PMS. Imagens a 30.000 qps.

5.25 Ignição e combustão no teste da mistura etanol e PEG400 (15\%), $1900 \mathrm{rpm}, \mathrm{RC}=25,58: 1$ e IDI $=7,00 \mathrm{~mm}\left(\sim 27^{\circ}\right.$ - Quadro 5) antes do PMS. Imagens a 30.000 qps.

5.26 Pressão no cilindro para o teste com a mistura de etanol e PEG400 (20\%), $1750 \mathrm{rpm}$ e $\mathrm{RC}=20,83: 1$.

5.27 Ignição e combustão no teste da mistura etanol e PEG400 (20\%), $1750 \mathrm{rpm}, \mathrm{RC}=20,83: 1$ e IDI $=4,00 \mathrm{~mm}\left(\sim 20^{\circ}\right.$ - Quadro 6) antes do PMS. Imagens a 30.000 qps.

5.28 Ignição e combustão no teste da mistura etanol e PEG400 (20\%), $1750 \mathrm{rpm}, \mathrm{RC}=20,83: 1$ e IDI $=5,60 \mathrm{~mm}\left(\sim 24^{\circ}\right.$ - Quadro 6) antes do PMS. Imagens a 30.000 qps.

5.29 Ignição e combustão no teste da mistura etanol e PEG400 (20\%), $1750 \mathrm{rpm}, \mathrm{RC}=20,83: 1$ e IDI $=7,20 \mathrm{~mm}\left(\sim 27^{\circ}\right.$ - Quadro 6) antes do PMS. Imagens a 30.000 qps.

5.30 Pressão no cilindro para o teste com a mistura de etanol e PEG600 $(7 \%), 1750 \mathrm{rpm}$ e $\mathrm{RC}=22,06: 1$.

5.31 Ignição e combustão no teste da mistura etanol e PEG600 (7\%), $1750 \mathrm{rpm}, \mathrm{RC}=22,06: 1 \mathrm{e}$ IDI $=5,20 \mathrm{~mm}\left(\sim 24^{\circ}-\right.$ Quadro 5) antes do PMS. Imagens a 30.000 qps.

5.32 Ignição e combustão no teste da mistura etanol e PEG600 (7\%), $1750 \mathrm{rpm}, \mathrm{RC}=22,06: 1$ e IDI $=6,60 \mathrm{~mm}\left(\sim 27^{\circ}\right.$ - Quadro 5) antes do PMS. Imagens a 30.000 qps.

5.33 Ignição e combustão no teste da mistura etanol e PEG600 (7\%), $1750 \mathrm{rpm}, \mathrm{RC}=22,06: 1$ e IDI $=8,50 \mathrm{~mm}\left(\sim 31^{\circ}\right.$ - Quadro 4$)$ antes do PMS. Imagens a 30.000 qps.

5.34 Pressão no cilindro para o teste com a mistura de etanol e PEG600 (7\%), $1900 \mathrm{rpm}$ e RC = 26,50:1.

5.35 Ignição e combustão no teste da mistura etanol e PEG600 (7\%), $1900 \mathrm{rpm}, \mathrm{RC}=26,50: 1$ e IDI $=6,50 \mathrm{~mm}\left(\sim 25^{\circ}\right.$ - Quadro 4) antes do PMS. Imagens a 30.000 qps.

5.36 Ignição e combustão no teste da mistura etanol e PEG600 (7\%), $1900 \mathrm{rpm}, \mathrm{RC}=26,50: 1$ e IDI $=8,60 \mathrm{~mm}\left(\sim 29^{\circ}\right.$ - Quadro 5) antes do PMS. Imagens a 30.000 qps. 
5.37 Ignição e combustão no teste da mistura etanol e PEG600 (7\%), $1900 \mathrm{rpm}, \mathrm{RC}=26,50: 1$ e IDI $=11,00 \mathrm{~mm}\left(\sim 33^{\circ}-\right.$ Quadro 4$)$ antes do PMS. Imagens a 30000 qps.

5.38 Pressão no cilindro para o teste com a mistura de etanol e n-Butanol $(10 \%), 1900 \mathrm{rpm}$ e RC $=26,81: 1$.

5.39 Ignição e combustão no teste da mistura etanol e n-Butanol (10\%), $1900 \mathrm{rpm}, \mathrm{RC}=26,81: 1$ e IDI $=5,70 \mathrm{~mm}\left(\sim 24^{\circ}\right.$ - Quadro 5) antes do PMS. Imagens a 30.000 qps.

5.40 Ignição e combustão no teste da mistura etanol e n-Butanol (10\%), $1900 \mathrm{rpm}, \mathrm{RC}=26,81: 1$ e IDI $=8,40 \mathrm{~mm}\left(\sim 29^{\circ}\right.$ - Quadro 4) antes do PMS. Imagens a 30000 qps.

5.41 Ignição e combustão no teste da mistura etanol e n-Butanol (10\%), $1900 \mathrm{rpm}, \mathrm{RC}=26,81: 1$ e IDI $=9,40 \mathrm{~mm}\left(\sim 31^{\circ}\right.$ - Quadro 4) antes do PMS. Imagens a 30.000 qps.

5.42 Pressão no cilindro para o teste com a mistura de etanol e n-Butanol $(15 \%), 1900 \mathrm{rpm}$ e RC $=26,81: 1$.

5.43 Ignição e combustão no teste da mistura etanol e n-Butanol (15\%), $1900 \mathrm{rpm}, \mathrm{RC}=26,81: 1$ e IDI $=7,50 \mathrm{~mm}\left(\sim 29^{\circ}\right.$ - Quadro 5) antes do PMS. Imagens a 30.000 qps.

5.44 Ignição e combustão no teste da mistura etanol e n-Butanol (15\%), $1900 \mathrm{rpm}, \mathrm{RC}=26,81: 1$ e IDI $=8,60 \mathrm{~mm}\left(\sim 31^{\circ}\right.$ - Quadro 4) antes do PMS. Imagens a 30.000 qps.

5.45 Ignição e combustão no teste da mistura etanol e n-Butanol (15\%), $1900 \mathrm{rpm}, \mathrm{RC}=26,81: 1$ e IDI $=9,70 \mathrm{~mm}\left(\sim 33^{\circ}\right.$ - Quadro 4$)$ antes do PMS. Imagens a 30.000 qps.

5.46 Pressão no cilindro para o teste com óleo Diesel S10, 1500 rpm e $\mathrm{RC}=16: 1$

5.47 Calor aparente liberado para o teste com óleo Diesel S10, 1500 rpm e RC $=16: 1$.

5.48 Pressão no cilindro para o teste com óleo Diesel S10, 1900 rpm e $\mathrm{RC}=20: 1$.

5.49 Calor aparente liberado para o teste com óleo Diesel S10, 1900 rpm e $\mathrm{RC}=20: 1$.

5.50 Pressão no cilindro para o teste com óleo Diesel S10, 2400 rpm e $\mathrm{TC}=24: 1$.

5.51 Calor aparente liberado para o teste com óleo Diesel S10, 2400 rpm e $\mathrm{RC}=24: 1$.

5.52 Pressão no cilindro para o teste da mistura de etanol e PEG 400 $(13 \%), 1900 \mathrm{rpm}$ e RC $=20: 1$.

5.53 Calor aparente liberado para o teste da mistura de etanol e PEG $400(13 \%), 1900 \mathrm{rpm}$ e RC $=20: 1$

5.54 Pressão no cilindro para o teste da mistura de etanol e PEG 400 $(13 \%), 2400 \mathrm{rpm}$ e RC $=24: 1$.

5.55 Calor aparente liberado para o teste da mistura de etanol e PEG $400(13 \%), 2400 \mathrm{rpm}$ e RC $=24: 1$

5.56 Pressão no cilindro para o teste da mistura de etanol e n-butanol $(10 \%), 2400 \mathrm{rpm}$ e $\mathrm{RC}=24: 1$. 
5.57 Calor aparente liberado para o teste da mistura de etanol e nbutanol (10\%), $2400 \mathrm{rpm}$ e RC $=24: 1$

5.58 Pressão no cilindro para o teste de dois combustíveis com IDI $=2$ $\mathrm{mm} \sim 13^{\circ}, 2400 \mathrm{rpm}$ e $\mathrm{RC}=24: 1$.

5.59 Pressão no cilindro para o teste de dois combustíveis com $|D|=3$ $\mathrm{mm} \sim 17^{\circ}, 2400 \mathrm{rpm}$ e $\mathrm{RC}=24: 1$. 


\section{Lista de tabelas}

2.1 Composição do Aditivo 54

2.2 Propriedades físicas e químicas dos compostos do ED95 55

2.3 Principais parâmetros analisados e método de ensaio empregado na caracterização do combustível ED95.

2.4 Valores de massa de etanol aditivado, resíduo obtido e \% final após os ensaios de destilação simples.

2.5 Valores de massa de etanol aditivado, resíduo obtido e \% final após os ensaios de destilação simples.

2.6 Viscosidade de alguns PEG a $100 \mathrm{C} \pm 0,3^{\circ} \mathrm{C}$.

2.7 Composição de combustível testado em motor de ignição por compressão

$\begin{array}{lll}2.8 & \text { Resultados obtidos para cada aditivo. } & 59\end{array}$

2.9 Dados sobre os isômeros do butanol 61

2.10 Propriedades dos combustíveis. 62

$\begin{array}{lll}3.1 & \text { Especificações da MCR } & 74\end{array}$

4.1 Poder calorífico inferior dos combustíveis testados. 88

4.2 Condições de operações para o teste de Diesel S50. 89

4.3 Condições de operações para teste com ED95. 90

4.4 Condições de operações para teste com Etanol + PEG400 (7\%). 90

4.5 Condições de operações para teste com Etanol + PEG400 (10\%). 90

4.6 Condições de operações para teste com Etanol + PEG400 (13\%). 91

4.7 Condições de operações para teste com Etanol + PEG400 (15\%). 91

4.8 Condições de operações para teste com Etanol + PEG400 (20\%). 92

4.9 Condições de operações para teste com Etanol + PEG600 (7\%). 92

4.10 Condições de operações para teste com Etanol + n-Butanol (10\%). 93

4.11 Condições de operações para teste com Etanol + n-Butanol (15\%). 93

4.12 Condições de operações para o teste de Diesel S10. 95

4.13 Condições de operações para teste com Etanol + PEG400 (13\%). 96

4.14 Condições de operações para teste com Etanol + n-Butanol (10\%). 96

4.15 Principais características técnicas do motor DW10CTED4 97

5.1 Resultados dos testes com Diesel S50 e ED95. 100

5.2 Resumo dos parâmetros da combustão para os combustíveis testados (Diesel S50 e ED95). 101

5.3 Resultados dos testes das misturas de etanol e PEG400. 110

5.4 Resumo dos parâmetros da combustão para as misturas de etanol e PEG400.

5.5 Resultados dos testes das misturas de etanol e PEG600. 126

5.6 Resumo dos parâmetros da combustão para as misturas de etanol e PEG600.

5.7 Resultados dos testes das misturas de etanol e n-Butanol.

5.8 Resumo dos parâmetros da combustão para as misturas de etanol e n-butanol. 
5.9 Resultados dos testes com Diesel S10. 142

5.10 Atraso da ignição para os testes com óleo Diesel S10. 149

5.11 Resultados dos testes da mistura de etanol e PEG 400 (13\%). 152

5.12 Atraso da ignição para os testes da mistura de etanol e PEG 400 $(13 \%)$.

5.13 Resultados dos testes da mistura de etanol e n-butanol (10\%). 159

5.14 Atraso da ignição para os testes da mistura de etanol e n-butanol $(10 \%)$.

5.15 Parâmetros da combustão para os diferentes combustíveis testados. 164

B.1 Incertezas dos equipamentos utilizados. 


\section{Lista de Símbolos}

\section{Símbolos Romanos}

$A \quad$ Constante para a determinação da relação $c_{p} / R$

$A / C \quad$ Razão ar - combustível

AEHC Álcool etílico hidratado combustível

AI Atraso da Ignição

ANP Agência Nacional do Petróleo, Gás Natural e Biocombustíveis

BEN Balanço Energético Nacional

${ }^{\circ} \mathrm{C} \quad$ Graus centígrados

c Calor específico

${ }^{\circ} \mathrm{CA} \quad$ Ângulo de virabrequim

CO Monóxido de carbono

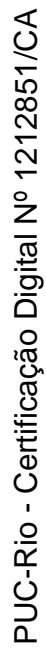

$\mathrm{CO}_{2} \quad$ Dióxido de carbono

$\mathrm{CH}_{3} \mathrm{OH}$ Metanol

$\mathrm{C}_{2} \mathrm{H}_{5} \mathrm{OH}$ Etanol

$d / d t \quad$ Primeira derivada em função do tempo

$d / d t^{2} \quad$ Segunda derivada em função do tempo

$d / d t^{3} \quad$ Terceira derivada em função do tempo

$D M \quad$ Desvio da média

$d Q / d t \quad$ Taxa de liberação de calor

E Inicio da injeção de combustível

EGR Sistema de recirculação dos gases de exaustão

fmq Fração de massa queimada

$h \quad$ Entalpia

$\mathrm{HC} \quad$ Hidrocarboneto

$\mathrm{H}_{2} \mathrm{O} \quad$ Água

ICE Ignição por centelha

ICO Ignição por compressão

ICOM Ignição por combustão

IDI Inicio da injeção

INPM Porcentagem de álcool em peso ou grau alcoólico

$J \quad$ Joule

$k J \quad$ Quilo Joule

L Variação na curva de pressão que indica o inicio da combustão

LEV Laboratório de Engenharia Veicular 


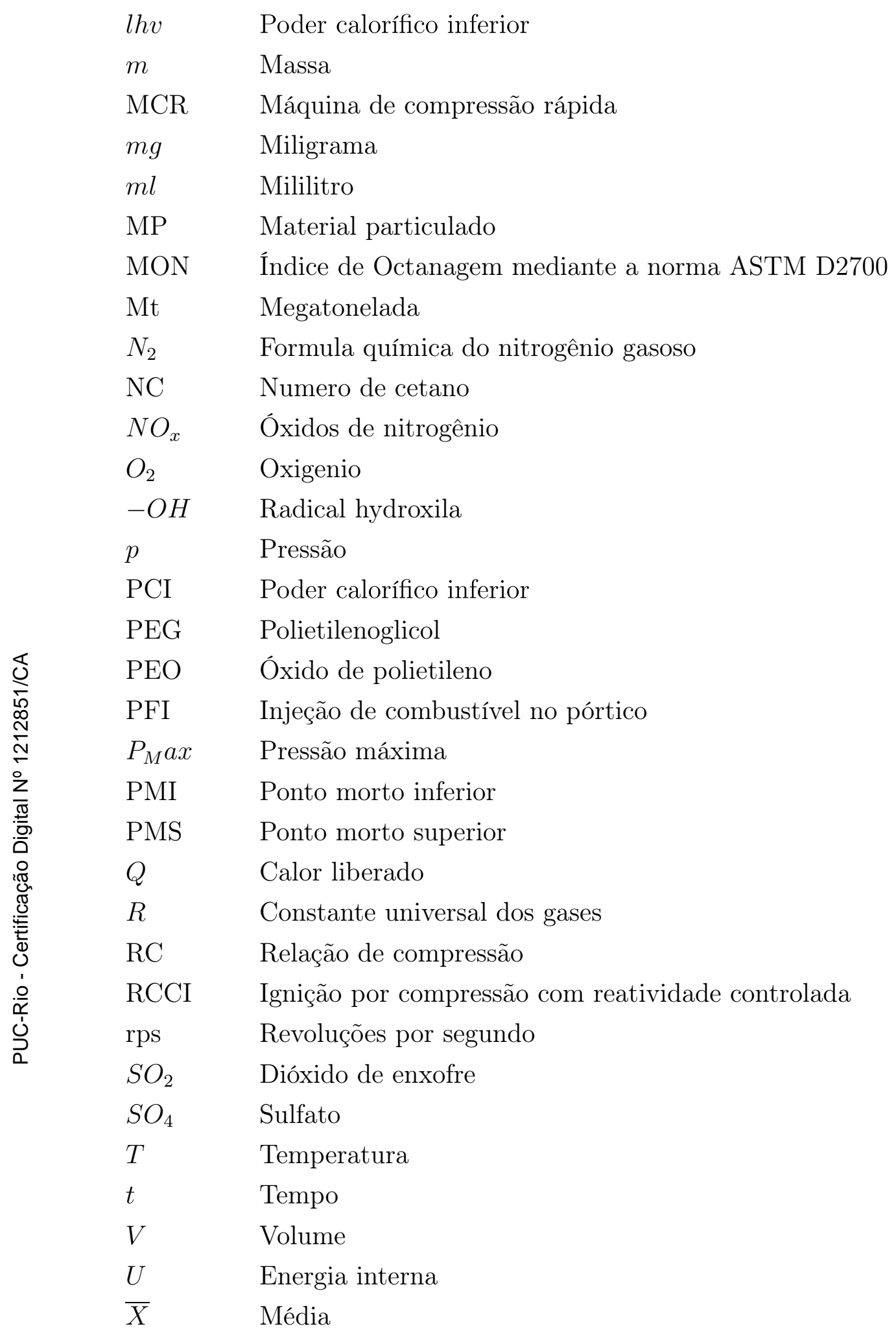

\section{Símbolos Gregos}

$\eta$ Eficiência

$\gamma$ Relação de calores específicos 


\section{Sub-índice}

$\begin{array}{ll}\text { ar } & \text { Ar } \\ c h & \text { Referente ao total calor liberado } \\ \text { comb } & \text { Combustível } \\ D & \text { Diesel } \\ e & \text { Etanol } \\ f & \text { Fluido } \\ h t & \text { Referente ao calor perdido pelas paredes } \\ i & \text { Instante de tempo } \\ n & \text { Referente ao calor líquido } \\ o & \text { Admissão de ar } \\ p & \text { Pressão constante } \\ v & \text { Volume constante }\end{array}$

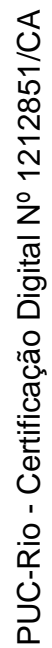

